\title{
Nonlinear study of injection process types into the traveling wave tube with hollow electron beam
}

\author{
H. Bayati ${ }^{1}$ - S. Saviz ${ }^{1}$ A. H. Sari ${ }^{1}$ \\ Received: 27 June 2018 / Accepted: 20 April 2019 / Published online: 10 May 2019 \\ (c) The Author(s) 2019
}

\begin{abstract}
In this paper, examples of all types of signal injection such as different signal, harmonic signal and multiple signal injections in a TWT including hollow electron beam near frequency $1800 \mathrm{MHz}$ and within input powers lower than TWT saturation power has been analyzed using approximate analytical solution of S-MUSE model. Later, the results obtained from approximate analytical solution with large signal code LATTE, that is a Lagrangian model, have been tested and simulated so that the effect of input phase difference between drive and injected wave and the effect of input powers on the amplification value of drive and generated second-order harmonic waves were investigated. The phase difference between drive and harmonic waves is a key parameter in harmonic injection for increasing the output power of drive and second-order harmonic frequencies.
\end{abstract}

Keywords Traveling wave tube (TWT) · Hollow electron beam · Different signal injections · Harmonic and multiple injections

\section{Introduction}

One of the major elements in a telecommunication transmitter is radio power amplifier. As regards in the new generations of wireless telecommunication, modulation is used, and considering the intensive changes of domain of transmitted signal in this modulation, linearity of power amplifier has a special importance, because nonlinearity of power amplifier in modulation causes lots of signal data and extension of its spectrum to the adjacent frequency bands $[1,2]$.

Nowadays, the traveling wave tube (TWT) in telecommunication systems and electronic war is used as a wave amplifier. Due to the nonlinear nature of this mean, a signal amplified by TWT undergoes distortion and it will attenuate the efficiency of mean and limit the bandwidth usable by amplifier. The distortion phenomenon in these systems causes the energy received by wave from electron beam to be transferred to the other second- and third-order harmonics which results in sound interference. Harmonic injection is method that is widely used for reduction in undesirable spectral content, because it results in reduction in sound inference

S. Saviz

azarabadegan@gmail.com

1 Plasma Physics Research Center, Science and Research Branch, Islamic Azad University, Tehran, Iran and makes the sound interference closer to a saturation level, and so increases the efficiency of amplifier [3-5].

Therefore, in this research the signal injection physics was studied for forming the output spectrum in a tube with traveling wave, using an analytical solution for approximating the nonlinear S-MUSE model with large signal code LATTE [6-8].

In TWT, a signal can transit along the circuit like as a transmission line. The circuit has been designed so that the signal velocity is almost similar to the velocity of electron beam transiting circuit. In addition, the electric fields arising from a signal on the circuit penetrate into the beam and cause interaction between wave and electron beam. The result of this interaction will be transfer of energy from electron beam to the wave and wave amplification [9-14].

There are three new TWT models with nonlinear fixed mode including MUSE, S-MUSE and LATTE models. These three models are suitable for study of nonlinear TWT phenomena. Lagrangian LATTE model has explained the most important nonlinear phenomenon such as RF wave power saturation and is suitable for simulation. MUSE model failed to predict the RF power saturation like as LATTE, and S-MUSE model removing the specific nonlinear conditions has been extracted from MSUE model and can be approximately before saturation considered as appropriate solution $[7,15,16]$. 


\section{Numerical results and discussion}

In order to study the role of cross section of hollow electron beam in a TWT, simulation was provided with large signal code LATTE. Large signal code LATTE is a onedimensional Lagrangian model that predicts the saturation of RF wave power saturation, carefully and well matched to the large signal code CHRISTINE 1-d and desirable for simulation [17-20].

In large signal code LATTE, a helix model is used as transmission line with loss and Lagrangian equations for electron beam $[10-14,16]$.

Here $\mathrm{z}$ is the axial distance, $\mathrm{t}$ is the time, $\mathrm{v}$ is the transmission line voltage, $\mathrm{I}$ is the transmission line current, $\mathrm{E}$ is the space charge electric field, $v$ is the electron beam velocity, $\Psi$ is the phase position of fluid element of an electron beam with the cross-sectional area $\mathrm{A}$, the dc electron beam velocity is $u_{0}, \mathrm{~L}$ is the inductance, $\mathbf{R}$ is the resistance, $\mathbf{C}$ is the capacitance, and $\mathrm{G}$ is the conductance. The functions $\mathrm{K}(\mathrm{z}, \mathrm{fl}), \mathrm{vph}(\mathrm{z}, \mathrm{fl})$ and $\mathrm{R}(\mathrm{z}, \mathrm{fl})$ are frequency domain circuit interaction impedance [12], cold circuit phase velocity and space charge reduction factor [11], respectively. The circuit equations, space charge equation, Newton's law and phase relation are [leaving off the superscript 1 ]. In this section, quantities with a tilde $(\sim)$ depend on frequency and axial distance $z$ [16].

$$
\begin{aligned}
\frac{\mathrm{d} V_{l}^{\sim}}{\mathrm{d} z}= & -i f_{l} \omega_{0} V_{l}^{\sim}+\left(\boldsymbol{R}_{l}^{\sim}-i f_{l} \omega_{0} L_{l}^{\sim}\right) I_{l}^{\sim} \\
\frac{\mathrm{d} I_{l}^{\sim}}{\mathrm{d} z}= & \left(G_{l}^{\sim}-i f_{l} \omega_{0} C_{l}^{\sim}\right) V_{l}^{\sim}-i f_{l} \omega_{0} I_{l}^{\sim} \\
& +i f_{l} \omega_{0} C_{l}^{\sim} \frac{\omega_{0}}{2 \pi} \int_{\frac{2 \pi}{\omega_{0}}} \frac{I_{0}\left(\psi_{0}\right) e^{-i f_{l} \omega_{0} \Psi\left(z, \psi_{0}\right)}}{I_{0} v\left(z, \psi_{0}\right)} \mathrm{d} \psi_{0} \\
\frac{\mathrm{d} E_{l}^{\sim}}{\mathrm{d} z}= & -i f_{l} \omega_{0} E_{l}^{\sim}+\frac{\omega_{0}}{2 \pi} \int_{\frac{2 \pi}{\omega_{0}}} \frac{I_{0}\left(\psi_{0}\right) e^{-i f_{l} \omega_{0} \Psi\left(z, \psi_{0}\right)}}{I_{0} v\left(z, \psi_{0}\right)} \mathrm{d} \psi_{0} \\
\frac{\partial v}{\partial z}= & \frac{1}{v} \sum_{l=-\infty}^{\infty}\left\{2 \boldsymbol{C}_{l}^{\sim 2}\left(i f_{l} \omega_{0} L_{l}^{\sim}-\boldsymbol{R}_{l}^{\sim}\right) I_{l}^{\sim}+R_{l}^{\sim} E_{l}^{\sim}\right\} e^{i f_{l} \omega_{0} \Psi\left(z, \psi_{0}\right)} \\
\frac{\partial \Psi}{\partial z}= & 1-\frac{1}{v}
\end{aligned}
$$

where the $f_{l}$ are integers indexed by $l$. The set of frequencies $\left\{f_{l}\right\}$ is chosen to be the set of frequencies with nonzero Fourier coefficients, and thus, $\left\{f_{l}\right\}$ is the set of drive frequencies together with the frequencies produced from nonlinear interactions. We index the frequencies so that $f_{-l}=-f_{l}$ and $f_{m}>f_{n}$ for $m>n$. Since our functions are real valued, $x_{-l}^{\sim}=x_{l}^{\sim *}($ Table 1$)$.

The calculations are done using a fixed-step fourthorder Runge-Kutta integrator. The circuit power at angular frequency $\omega$ is due to both the positive and negative frequencies [15] and so

$P_{\omega}(z)=-\left[V_{l}^{\sim}(z) I_{l}^{\sim *}(z)+V_{l}^{\sim *}(z) I_{l}^{\sim}(z)\right]$

The negative sign is due to the form of the telegrapher equations which are chosen to be consistent with [10]. We simulate one constant pitch section of the $8537 \mathrm{H}$ TWT with sever or circuit loss. The parameters for the $8537 \mathrm{H}$ are taken from [19] and are shown in Table 2 for the freq u e n c y-d e pende n t parame ter s $\nu_{p h}^{\sim}\left(f_{l} \omega_{0}\right), K^{\sim}\left(f_{l} \omega_{0}\right)$, and $R^{\sim}\left(f_{l} \omega_{0}\right)$. We use the outputs of CHRISTINE 1-d's tape helix model and space charge reduction factor calculation, as shown in Table 3. These parameter values ensure that LATTE uses the same dispersion parameters as CHRISTINE 1-d.

In this paper, a general "signal injection" theory is explained using approximate analytical solutions of S-MUSE model. The approximate solution consists of injected terms and generated terms that are created by natural nonlinear processes of wave upon interaction to the hollow electron beam. When required, the results obtained from approximate analytical solution are tested with large signal code LATTE. LATTE has been derived from the same initial S-MUSE equations and therefore was suitable ideally for this purpose. Analytical solution for S-MUSE has infinite modes. To form the approximate solutions,

Table 2 8537H parameters (constant pitch section)

\begin{tabular}{ll}
\hline Parameter & Model value \\
\hline Helix mean radius & $0.2353 \mathrm{~cm}$ \\
Helix wire width & $0.0305 \mathrm{~cm}$ \\
Pitch & $0.13 \mathrm{~cm}$ \\
Cathode voltage & $-3.1 \mathrm{kV}$ \\
Beam current & $65.5 \mathrm{~mA}$ \\
Min. beam radius & $0.0962 \mathrm{~cm}$ \\
$\mathrm{BN}\left(\epsilon_{r}=5.4\right)$ Smeared permittivity & 1.21 \\
\hline
\end{tabular}

Table 1 Sever position with the amount of loss in terms of the axial distance in length of helix

\begin{tabular}{llllllll}
\hline Loss-location $(\mathrm{cm})$ & 0.0 & 7.0 & 12.0 & 12.5 & 20.0 & 24.0 & 30.0 \\
Circuit attenuation $(\mathrm{dB} / \mathrm{cm})$ & 0.2 & 0.2 & 1.0 & 30.0 & 30.0 & 1.0 & 0.2 \\
\hline
\end{tabular}


Table 3 Simulation frequencies and dispersion parameters

\begin{tabular}{llll}
\hline$f(\mathrm{GHz})$ & $K^{\sim} \Omega$ & $v_{p h}^{\sim}\left(\times 10^{7} \mathrm{~m} / \mathrm{s}\right)$ & $R^{\sim}$ \\
\hline 0.001 & 365.40 & 3.2845 & 0.00100 \\
1.599 & 225.13 & 2.9983 & 0.04529 \\
1.600 & 224.98 & 2.9980 & 0.04535 \\
1.601 & 224.82 & 2.9977 & 0.04540 \\
1.602 & 224.67 & 2.9974 & 0.04545 \\
3.200 & 42.68 & 2.6460 & 0.14945 \\
3.201 & 42.62 & 2.6459 & 0.14952 \\
3.202 & 42.57 & 2.6458 & 0.14959 \\
\hline
\end{tabular}

finite modes are used. Moreover, when required, only those modes are considered that are ruling on solution near the TWT output which are common modes with largest growth speed. Besides, several cases of signal injection are investigated and studied. Firstly, different signal and later second-order harmonic signal injections are considered for neutralization of harmonic and promotion of derive frequency. In this study, the effect of multiple harmonic injections has been applied.

The structure of the S-MUSE solution for a physical variable at a particular frequency is a sum of complex exponential modes. In general, voltage solving in frequency $f=f_{l} \omega_{0}$ may be approximated as follows:

$$
\begin{aligned}
V_{l}(z, t)= & \left\{A_{l}^{d r} \exp \left(\mu_{l}^{d r}+i k_{l}^{d r}\right) z+\sum_{q} A_{l}^{n l[q]}\right. \\
& \left.\times \exp \left(\mu_{l}^{n l[q]}+i k_{l}^{n l[q]}\right) z\right\} \times e^{i f_{l} \omega_{0}\left(\left(z / u_{0}\right)-t\right)}
\end{aligned}
$$

Therein, upper index dr denotes driven values and upper index $\mathrm{nl}$ denotes values generated by nonlinear interactions, also $\mu$ is growth rates, $k$ is phase angles in (7), and these are together as complex propagation constants. The idea of all injection methods is setting and adjusting the domains and input phases so that the sentence in bracket of Eq. (7) reaches to its minimum in $z=L$, therein $L$ denotes TWT output. In some parts of this paper that describes different designs of injection, the structure of different elements of Eq. (7) will be identified $[6,7]$.

\section{Results of Simulations}

In this paper, at first TWT model $8537 \mathrm{H}$ (Hughes) that has been made industrially and used helix as SWS was studied and modeled in consideration of attenuation in wave amplifying circuit as in Fig. 1, by large signal code LATTE, and its output power was calculated in frequencies 1500-2100 MHz. As observed, this wave amplifier within

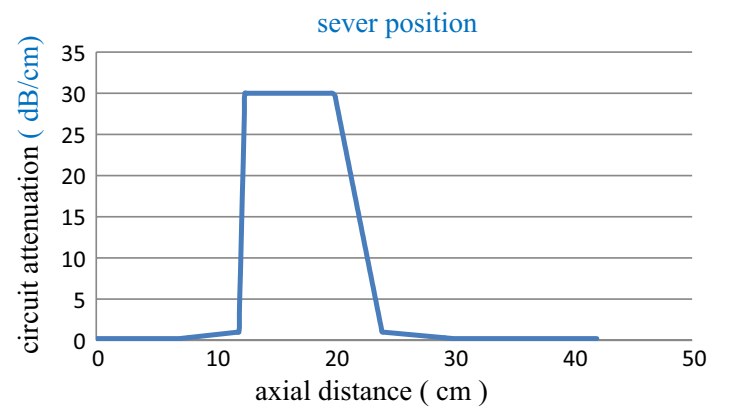

Fig. 1 TWT circuit attenuation based on the axial distance

the frequency $1800 \mathrm{MHz}$ has the maximum efficiency and output power.

Because of the internal modulation and the production of the second- and third-order harmonics in the TWT, the power and energy decrease at the original frequency, and also due to the formation of reciprocating or retrogressive waves and the interference of these waves with the original wave, the helix fluctuates and warms up, which causes the loss of energy and attenuation in the system. Of course, the change in the helix step is a way to control the fluctuation of the return wave. Certainly, all TWTs have circuit severs to control internal reflections. In this paper, we considered the circuit sever and Table 1 shows the sever position with the amount of loss in terms of the axial distance in length of helix, which resulted in the modeling of Fig. 1.

For the frequencies not listed in Table 3, the parameters of propagation (cold circuit phase velocity, the interaction impedance and space charge reduction factor) are determined by a linear interpolation among adjacent frequencies. The TWT helix's length is $L=42 \mathrm{~cm}$. Almost all of TWTs have circuit severs to control the internal reflections. We consider circuit sever that is modeled by a resistant loss in the central section of the circuit as per Fig. 1.

To calculate the power gain, following equation was used [21]:

Power gain $=10 * \log \mid$ Pout $/$ Pin $\mid$

Reasonable adaption of simulation results to the experimental test results not only confirms the correct simulation trend, but also will be a mean for validation and remedy of the defects of analytical design process. The input and output power curves of a TWTA have been often normalized for an output power. Instead, considering the real input power to watt or $\mathrm{dbm}$, the curve has been normalized so that produces $0 \mathrm{db}$ in saturation state.

At the first phase of this research, a mode of cross section is considered for hollow electron beam so that its output radius Rout $=0.09652 \mathrm{~cm}$ and its input radius is $\operatorname{Rin}=0.08686 \mathrm{~cm}$ that is $90 \%$ of output radius of electron beam and has a cross-sectional area of 
$A=5.561 \times 10^{-3}\left(\mathrm{~cm}^{2}\right)$ and input current density is $\rho_{\circ}=3569 \times 10^{-12\left(\mathrm{c} / \mathrm{cm}^{3}\right)}$. In this mode, seven sine waves with frequencies $1500 \mathrm{MHz}, 1600 \mathrm{MHz}, 1700 \mathrm{MHz}$, $1800 \mathrm{MHz}, 1900 \mathrm{MHz}, 2000 \mathrm{MHz}$ and $2100 \mathrm{MHz}$ and with input power Pin $=-10 \mathrm{dbm}$ and input phases $\phi$ in $=90.0^{\circ}$ are entered into the TWT structure singly. After interaction of each wave to the hollow electron beam through simulation, the output power of wave and its output phase is obtained, as diagram of their output power and output voltage phase based on the axial distance $\mathrm{z}$ is shown in Figs. 2 and 3.

As observed in Figs. 2 and 3, in the aforesaid conditions within frequency $1500-1900 \mathrm{MHz}$, the phase changes along TWT are mild. Besides, within frequency range $1700-1900 \mathrm{MHz}$, the output power is in its climax and the power gain of TWT is maximum. Therefore, in continue of research, we work on frequencies close to $1800 \mathrm{MHz}$.

\section{Injection of different signals}

In the second phase of this paper, different signal injections are considered so that the drive signal with frequency $F 1=1800 \mathrm{MHz}$, input power Pin $=-10 \mathrm{dbm}$ and phase $\phi i n=0.0^{\circ}$," injection signal with frequency $F 2=1799 \mathrm{MHz}$ and input power Pin $=-10 \mathrm{dbm}$ and input phase is $\phi o u t$, so that oout $=0,10,20,30, \ldots, 360$ degrees simultaneously enter into TWT structure, and after interaction to the hollow electron beam, through simulation, the output phase and power of produced drive frequencies and second-order harmonics that situated within linear bandwidth were examined. Second-order harmonics include frequencies $2 F 2=3598 \mathrm{MHz}$, $2 F 1=3600 \mathrm{MHz}, F 1+F 2=3599 \mathrm{MHz}, F 1-F 2=1 \mathrm{MHz}$. However, frequency $F 1-F 2=1 \mathrm{MHz}$ is situated out of linear bandwidth that is ignored. Therefore, two frequencies $1799 \mathrm{MHz}$ and $1800 \mathrm{MHz}$ are two drive frequencies, and three frequencies including $3598 \mathrm{MHz}, 3599 \mathrm{MHz}$ and $3600 \mathrm{MHz}$ are second-order harmonic frequencies. Upon ignorance of third-order intermodulation products, the response of voltage (7) for frequency $1800 \mathrm{MHz}$ is a driven mode (the same $1800 \mathrm{MHz}$ ), and two modes are nonlinear. First nonlinear mode exists in $3599 \mathrm{MHz}$, because $3599 \mathrm{MHz}$ is sum of frequencies $1799 \mathrm{MHz}$ and $1800 \mathrm{MHz}$, and second nonlinear mode exists in $3600 \mathrm{MHz}$, because $3600 \mathrm{MHz}$ is the product of second-order harmonic of $1800 \mathrm{MHz}$. Moreover, for the drive frequency $1799 \mathrm{MHz}$, there is a driven mode (the same $1799 \mathrm{MHz}$ ) and two nonlinear modes. First nonlinear mode exists in $3599 \mathrm{MHz}$, because $3599 \mathrm{MHz}$ is sum of frequencies $1799 \mathrm{MHz}$ and $1800 \mathrm{MHz}$, and second nonlinear mode exists in $3598 \mathrm{MHz}$, because $3598 \mathrm{MHz}$ is the product of secondorder harmonic of $1799 \mathrm{MHz}$. After simulation of this array with large signal code LATTE, the output phases and powers
Fig. 2 Output power based on axial distance for seven sine waves with varied frequencies and equal input powers Pin $=-10 \mathrm{dbm}$ and equal input voltage phase $\phi$ in $=90.0^{\circ}$ that entered singly into TWT structure
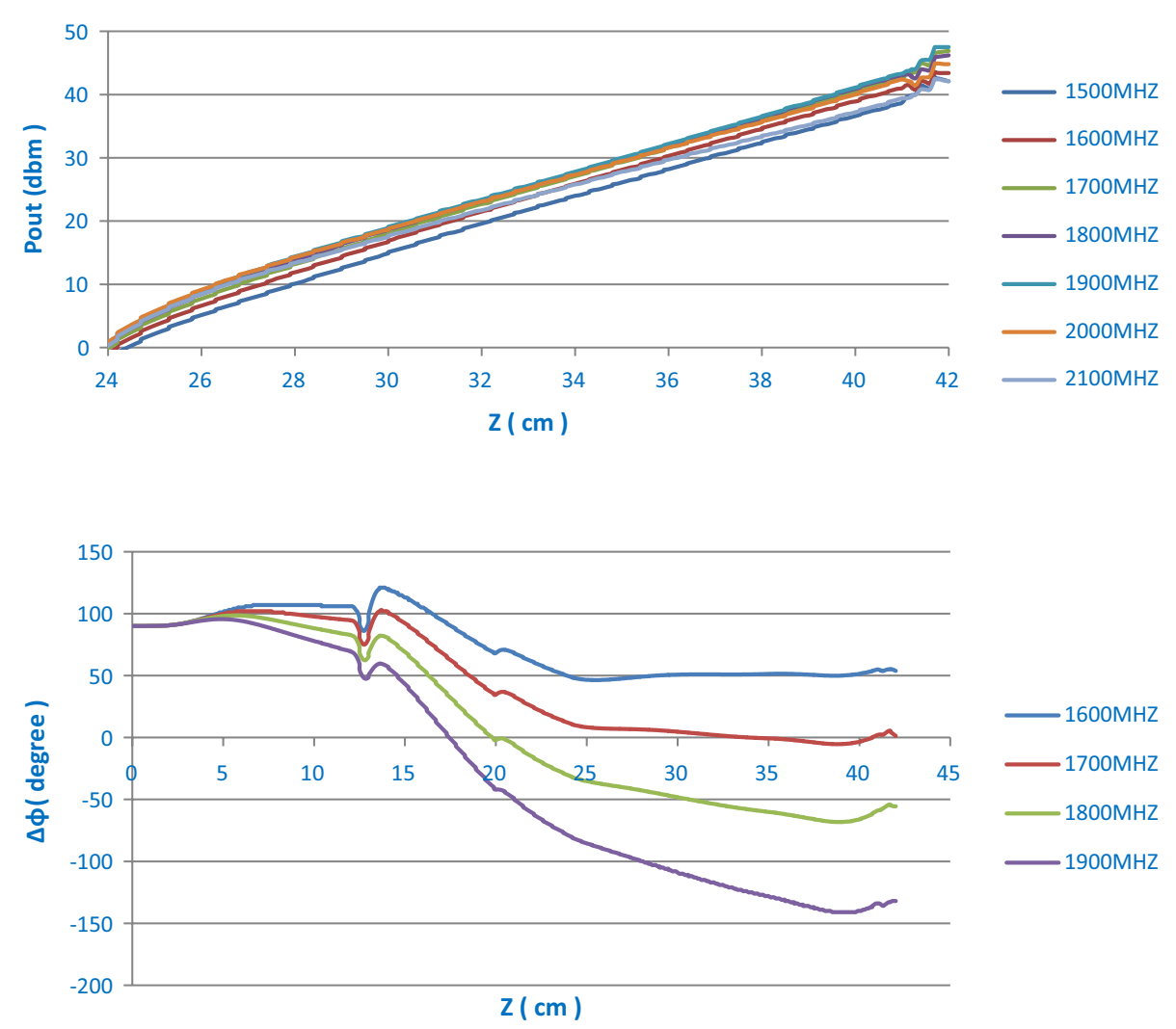

Fig. 3 Variations of voltage phase based on axial distance for four sine waves with varied frequencies and equal input powers $P$ in $=-10 \mathrm{dbm}$ and equal input voltage phase $\phi i n=90.0^{\circ}$ that entered singly into TWT structure 


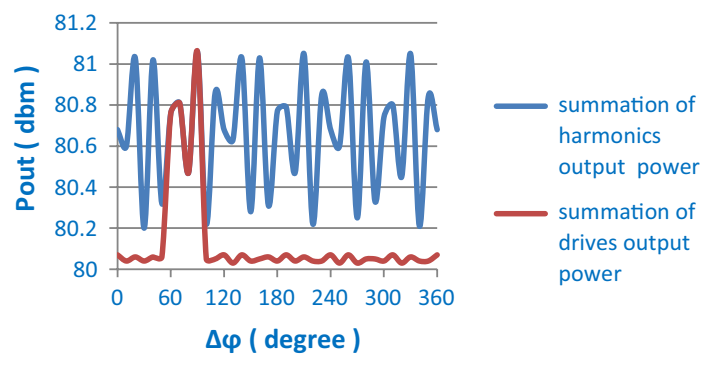

Fig. 4 Diagram of summation of output powers of drives and summation of output powers of second-order harmonic frequencies based on the difference of input phase of two drive frequencies so that the drive signal with frequency $1800 \mathrm{MHz}$, input power $-10 \mathrm{dbm}$, input phase $\phi i n=0.0^{\circ}$ and injection signal with frequency $1799 \mathrm{MHz}$, input power $-10 \mathrm{dbm}$ and varied input phase

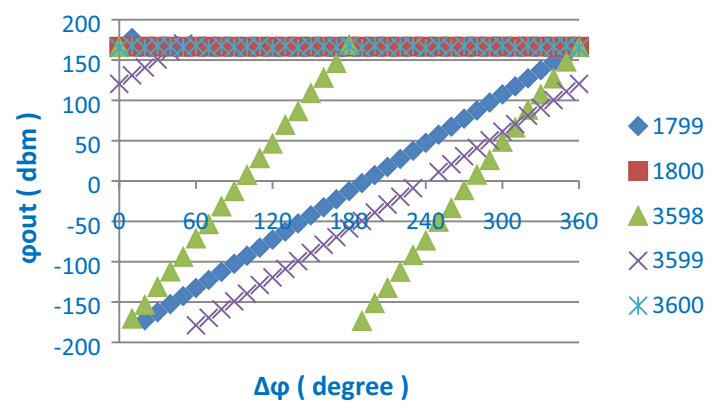

Fig. 5 Diagram of output voltage phase of drive and second-order harmonic frequencies based on the difference of input phase of two drive frequencies so that the drive signal with frequency $1800 \mathrm{MHz}$, input power $-10 \mathrm{dbm}$, input phase $\phi i n=0.0^{\circ}$ and injection signal with frequency $1799 \mathrm{MHz}$, input power -10 dbm and varied input phase

of drive and second-order harmonic signals are measured and summation of output powers of drive and second-order harmonic frequencies that are connected to the energy taken by waves from hollow electron beam is compared and the related diagram is shown in Figs. 4 and 5.

As observed in Fig. 4, the difference of input phase between drive and injection wave is effective on the interaction of wave to the electron beam and value of energy transmitted from electron beam to the wave, and also affects the drives and second-order harmonics amplification value, so that in the difference of specific phases, the energy transferred to the second-order harmonics is maximum, while the energy transferred to the drive waves is normal, and only if the phase difference becomes $90.0^{\circ}$, the energy arrives to the drive signals and total second-order harmonics is maximum that is the result of maximum energy transfer from electron beam.

As observed in Fig. 5, the difference of input phase of drive signals and injection signals has no impact on the output phase of drive signal, and only affects the output phase of injection signal and second-order harmonics.

\section{Harmonic signal injection}

In the third phase of this paper, second-order harmonic injection at the presence of a drive frequency is considered. The injected second-order harmonic on the drive and harmonic frequencies is studied and analyzed. A drive wave with frequency $F=1800 \mathrm{MHz}$, input power Pin $=-10 \mathrm{dbm}$ and phase $\phi$ in $=0.0^{\circ}$ enters into the TWT structure. At this time, the second-order harmonic of wave with frequency $2 F=3600 \mathrm{MHz}$ is injected in-phase with the drive wave. According to the interaction between drive signal and injection signal with hollow electron beam, analytically it is concluded that the response of voltage (7) for each one of these frequencies is a driven and a nonlinear mode. There is a nonlinear mode in $1800 \mathrm{MHz}$, because $1800 \mathrm{MHz}$ is difference between frequency $3600 \mathrm{MHz}$ and $1800 \mathrm{MHz}$. Nonlinear mode exists in $3600 \mathrm{MHz}$ of second-order harmonic $1800 \mathrm{MHz}$. After simulation of the above structure, the output phase and power of produced drive and second-order harmonic wave are examined. As concluded from Table 4, upon varying the input power of injected harmonic into a constant phase, the output power level of drive signal is not changed and reaches to the saturation state. In other word, the input power of injected harmonic has no effect on the output power of drive frequency. Besides, according to Fig. 6 if the input phase of injected second-order harmonic is changed, while its input power is constant, again it has no impact on the output power of drive and second-order harmonic frequency. Note that in this paper, the level of input powers is lower than saturation level. Perhaps, if the level of input powers is higher than saturation level, different results are obtained.

\section{Multiple signal injection}

In the fourth phase of this research, a combination of varied frequency injection and harmonic frequency injection is considered that is a sample of multiple injections. Two drive signals with $F 1=1800 \mathrm{MHz}$ and $F 2=1799 \mathrm{MHz}$ and equal powers enter into the TWT structure. If at this moment, second-order harmonic of both drive signals with $2 F 1=3600 \mathrm{MHz}$ and $2 F 2=3598 \mathrm{MHz}$ sizes is injected into

Table 4 Output power of drive frequency based on input power of injected harmonic

\begin{tabular}{llllll}
\hline Input power of injected harmonic $(\mathrm{dbm})$ & -5 & -10 & -15 & -20 & -25 \\
Output power of drive frequency $(\mathrm{dbm})$ & 44.96 & 44.96 & 44.96 & 44.96 & 44.96 \\
\hline
\end{tabular}




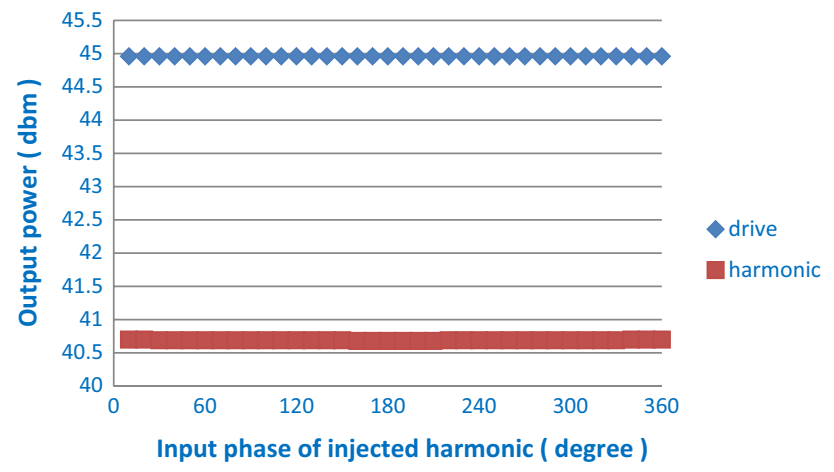

Fig. 6 Output power of drive and harmonic frequencies based on input phase of injected harmonic so that driver frequency $(f=1800 \mathrm{MHz})$ with input phase $0.0^{\circ}$ and input power $-10 \mathrm{dbm}$ and a harmonic injected frequency $(f=3600 \mathrm{MHz})$ with input power $-10 \mathrm{dbm}$ and input phase $\phi i n$, so that $\phi i n=10.0^{\circ}, 20.0^{\circ}, \ldots, 360.0^{\circ}$

the TWT, the input powers of drive and second-order harmonic signals are equal and $\mathrm{Pin}=-10 \mathrm{dbm}$, the input phase $F 1$ and $2 F 1$ is equal to $0.0^{\circ}$, and the input phase $F 2$ is equal to $2 F 2$, but varied, so that after interaction and simulation, the output frequency spectrum is observed as 1798, 1799, 1800, 1801, 3598, 3599, $3600 \mathrm{MHz}$. Upon measuring the output powers of produced drive and second-order harmonic waves, it is said if two drive waves have phase difference $0^{\circ}, 60^{\circ}, 120^{\circ}, 180^{\circ}, 240^{\circ}, 300^{\circ}, 360^{\circ}$, summation of output powers of drive frequencies will be maximum, and in case two drive waves have phase difference $40^{\circ}, 70^{\circ}, 100^{\circ}, 160^{\circ}$, $190^{\circ}, 220^{\circ}, 280^{\circ}, 310^{\circ}, 340^{\circ}$, summation of output powers of drive frequencies is minimum, and in return, summation of output powers of frequencies of driven and produced second-order harmonic frequencies will be maximum. In $120^{\circ}$, largest maximum value of summation of output powers of drive frequencies is obtained, and in $40^{\circ}$, maximum summation of output powers of driven and produced second-order frequencies is obtained. Therefore, in general when the level of driven and injected input powers is lower than saturation power level, the phase difference may be effective on the energy transfer to the waves, while if the powers level is about the saturation level or higher, the role of phase difference is faded more.

Now, the output spectrums with and without injection of the both second harmonics are shown in Figs. 7 and 8.

As observed in Figs. 7 and 8, in mode without injection of second harmonics of both drive frequencies, there is no extra signal near the drive frequencies, but in mode with injection of second harmonics of the both drive frequencies, some spectral elements of third-order intermodulation such as $f=1798 \mathrm{MHz}$ and $f=1801 \mathrm{MHz}$ have been appeared near drive frequencies. However, the power level of these extra signals is lower than power of drive frequencies, and the higher this difference of output power level, the more desirable in better efficiency of TWTs. Reaching to the better conditions is subject to investigation and research in the future.

\section{Results}

In this paper, examples of all types of signal injection including different signal, harmonic signal and multiple signal injections in a TWT was modeled and analyzed.

1. If in different signal injections, the frequency difference between two input waves is $1 \mathrm{MHz}$ and input power level is lower than saturation power level, the input phase difference between drive and injected wave is effective on the wave interaction to the electron beam and energy transferred from electron beam to the wave, and affects the magnitude of the amplification of the drive and produced second-order harmonic waves so that it is maximum in the specific phase differences and that energy is transferred to the second-order harmonics, while the energy transferred to the drive waves is normal, and if only the phase difference is $90.0^{\circ}$, the energy transferred to the drive and total second-order harmonic signals is

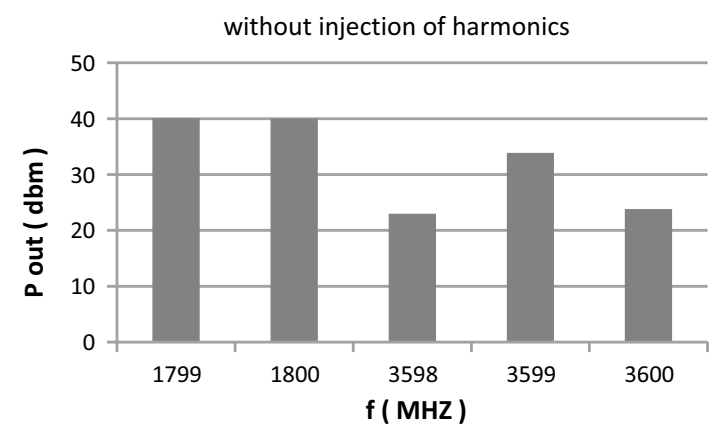

Fig. 7 Spectrum of output signals in different frequency injection states

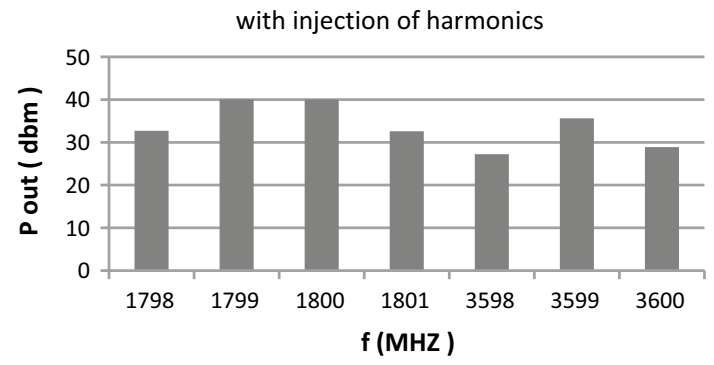

Fig. 8 Spectrum of output signals in different frequency injections together with harmonic injection. Notice the additional frequencies caused by the injection of these signals. The inputs of drive frequencies 1800 and $1799 \mathrm{MHz}$ have input power $-10 \mathrm{dbm}$ and input phases $0.0^{\circ}$. The injected second harmonic frequency $3600 \mathrm{MHz}$ and $3598 \mathrm{MHz}$ has input power $-10 \mathrm{dbm}$ and input phases $0.0^{\circ}$ 
maximum, which is the result of maximum energy transfer from electron beam. In addition, the difference of input phase of drive and injected signals has no effect on the output phase of drive signal and only affects the output phase of injected and second-order harmonic signal.

2. If the second-order harmonic signal injection is made at the presence of a drive frequency in-phase, and the input power level is lower than the saturation power, upon changing the input power of injected harmonic in a constant phase, the output power of drive signal is not varied and reaches to the saturation state. In other word, the input power of injected harmonic has no impact on the output power of drive frequency. Moreover, if the input phase of injected second-order harmonic is changed, while its input power is constant, it has no impact on the output power of drive and second-order frequency. Note that in this paper, the input powers level is lower than saturation level. Perhaps, if the input power level is higher than saturation level, the different results will be obtained.

3. If in multiple signal injection that is a combination of different frequency and harmonic frequency injections, and the difference of frequency of two drive waves is $1 \mathrm{MHz}$ and second-order harmonic $(2 F)$ of both drive signals is injected, the input powers of drive and injected second-order harmonic signals are equal and lower than power saturation, and the input phase of each drive signal is equal to its doubled frequency, and if two drive waves have a phase difference of $120^{\circ}$, summation of output powers of drive frequencies is maximized, and if two drive waves have a phase difference of $40^{\circ}$, summation of output powers of driven and produced secondorder harmonic frequencies is maximized, and the phase difference may be effective on the energy transfer to the waves; while if the powers level is about saturation level or higher, the role of phase difference is faded. In without injection state, the second harmonics of every drive frequencies, no extra signal exists within the drive frequencies, but in with injection state, the second harmonics of the both drive frequencies, some spectral elements of third-order intermodulation with lower power level than power level of drive frequencies, appeared around the drive frequencies.

Open Access This article is distributed under the terms of the Creative Commons Attribution 4.0 International License (http://creativeco mmons.org/licenses/by/4.0/), which permits unrestricted use, distribution, and reproduction in any medium, provided you give appropriate credit to the original author(s) and the source, provide a link to the Creative Commons license, and indicate if changes were made.

\section{References}

1. Ramo, S., Whinnery, J.R., Van Duzer, T.: Fields and Waves in Communication Electronics. Wiley, New York (1965)

2. Calame, J.P., Gray, H.F., Shaw, J.L.: Analysis and design of microwave amplifiers employing field emitter arrays. J. Appl. Phys. 73, 1485-1504 (1993)

3. Wirth, M., Singh, A., Scharer, J., Booske, J.: Third-order intermodulation reduction by harmonic injection in a TWT amplifier. IEEE Trans. Electron. Devices 49, 1082-1084 (2002)

4. Kalkanli, Guzelyurt, Mersin: Impact of second harmonic injection on the linearity and linear gain of RF/microwave amplifiers. Int. Electron 100(1), 72-93 (2013)

5. Dani, A., Roberg, M., Popovic, Z.: PA efficiency and linearity enhancement using external harmonic injection. IEEE Trans. Microw. Theory Tech. 60(12) (2012)

6. Wohlbier, J.G., Booske, J.H., Dobson, I.: On the physics of harmonic injection in a traveling wave tube. IEEE Trans. Plasma Sci. 32(3), 1073-1085 (2004)

7. Wöhlbier, J.G., Dobson, I., Booske, J.H.: Generation and growth rates of nonlinear distortions in a traveling wave tube. Phys. Rev. E 66(5), 056504 (2002)

8. Plouin, J., Rax, J.M., Andre, F., Thaler, Y.: Improvement of the output power in TWTs by harmonic injection. IEEE Transactions on Electron Devices 52(5), 783-789 (2005)

9. Zhuge, T., Hu, Y.: Design of a Novel high power V-band helixfolded waveguide cascaded traveling wave tube amplifier. Hindawi Pupli. 2015, 846425

10. Gilmour Jr., A.S.: Principles of Traveling Wave Tubes. Artech House, Boston (1994)

11. Hutter, R.G.E.: Beam and Wave Electronics in Microwave Tubes. D.Van Nostrand Company, Inc, Princeton (1960)

12. Gewartowski, J.W., Watson, H.A.: Principles of Electron Tubes. D. Van Nostrand Company, Inc, Princeton (1965)

13. David, D.K.: Field and wave Electromagnetics, 2nd edn. AddisonWesley Publishing Co., Reading (1989)

14. Pierce, J.R.: Traveling Wave Tubes. Van Nostrand, Princeton (1950)

15. Wohlbier, J.G., Booske, J.H., Dobson, I.: The multifrequency spectral Eulerian (MUSE) model of a traveling wave tube. IEEE Trans. Plasma Sci. 30(3), 1063-1075 (2002)

16. Wohlbier, J.G.: Nonlinear distortion and suppression in traveling wave tube: insights and methods. Ph.D. dissertation, Univ. Wisconsin-Madison (2003)

17. Antonsen Jr., T.M., Levush, B.: Traveling-wave tube devices with nonlinear dielectric elements. IEEE Trans. Plasma Sci. 26(3), 774-786 (1998)

18. David, J.A., Kory, C.L., Tran, H.T., Ives, R.L., Chernin, D.: Enhanced features for design of traveling wave tubes using CHRISTINE-1D. IEEE Trans. Plasma Sci. 35(4), 1056-1064 (2007)

19. Abe, D.K., Ngo, M.T., Levush, B., Antonsen Jr., T.M., Chernin, D.P.: A comparison of L-band helix TWT experiments with CHRISTINE, a 1-D multi-frequency helix TWT code. IEEE Trans. Plasma Sci. 28(3), 576-587 (2000)

20. Antonsen, T.M., Jr, Levush, B.: CHRISTINE: A multi-frequency parametric simulation code for traveling wave tube amplifiers. NRL Memo report NRL/FR/6840-97-9845 (1997)

21. Sedra, A., Smith, K.C.: Microelectronic Circuits. Oxford University, Oxford (1998)

Publisher's Note Springer Nature remains neutral with regard to jurisdictional claims in published maps and institutional affiliations. 